\title{
Le retour au prisme de ses détours ou comment réintroduire de la proximité dans l'éloignement
}

Constance De Gourcy

\section{OpenEdition}

1 Journals

Édition électronique

URL : https://journals.openedition.org/remi/4184

DOI : $10.4000 /$ remi.4184

ISSN : $1777-5418$

Éditeur

Université de Poitiers

Édition imprimée

Date de publication : 1 octobre 2007

Pagination : 159-171

ISBN : 978-2-911627-46-6

ISSN : 0765-0752

\section{Référence électronique}

Constance De Gourcy, «Le retour au prisme de ses détours ou comment réintroduire de la proximité dans l'éloignement », Revue européenne des migrations internationales [En ligne], vol. 23 - n² | 2007, mis en ligne le 01 octobre 2010, consulté le 16 avril 2022. URL : http://journals.openedition.org/remi/4184 ; DOl : https://doi.org/10.4000/remi.4184

Ce document a été généré automatiquement le 16 avril 2022

() Université de Poitiers 


\title{
Le retour au prisme de ses détours ou comment réintroduire de la proximité dans l'éloignement
}

\author{
Constance De Gourcy
}

\begin{abstract}
«Plus vaste est le temps que nous avons laissé derrière nous, plus irrésistible est la voix qui nous invite au retour. Cette sentence a l'air d'une évidence, et pourtant elle est fausse » (Kundera,
\end{abstract}

2003).

1 Lorsqu'il s'agit du retour, on pense, avant tout, à la dimension quasi exclusivement géographique qui le sous-tend. On considère, en effet, généralement qu'il y a retour quand une personne revient physiquement sur les lieux d'où elle est partie à un moment donné - même si la période peut être plus ou moins longue selon les cas considérés ${ }^{1}$. Le retour traduit ainsi l'idée d'un déplacement qui s'exprime en sens inverse d'un mouvement précédent.

2 Or, lorsqu'il est analysé à l'aune de parcours migratoires, d'autres possibles apparaissent en filigrane. Les qualités d'habitabilité et d'hospitalité investies dans les lieux de destination brouillent les repères et invitent à considérer autrement le rapport entre lieux de départ et lieux de destination. Dès lors, le retour ne se saisit plus simplement comme un procès de rebroussement.

3 Pour illustrer cette conception "élargie " du retour et les incidences que cela peut avoir, notamment sur la notion d'inscription territoriale, notre réflexion portera sur une population de migrants rencontrés dans le cadre d'une recherche portant sur l'autonomie dans la migration (De Gourcy, 2005). Des migrants qui disaient s'être installés dans des villes de destination, retenues comme points de convergence de leurs parcours migratoires ${ }^{2}$, sans que des raisons professionnelles, matérielles, familiales, politiques ou autres aient été en premier lieu à l'origine de leur départ. Certains disaient même avoir réalisé des sacrifices (en renonçant par exemple à un travail mieux rémunéré, en s'éloignant de leur cercle familial) pour mettre en œuvre cette migration. 
La plupart venaient de pays lointains (Australie, Japon,...), d'autres de pays plus proches (Espagne, Algérie, Tunisie...) ; mais, quel que soit leur pays de départ, tous quittaient un environnement connu pour aller vers l'inconnu, tous s'affranchissaient d'un certain ordre pour le désordre auquel les renvoyait leur décision.

Exprimant un désengagement à l'égard des déterminismes matériels et sociaux, ces migrants volontaires ${ }^{3}$, population non représentée par les compteurs statistiques mais représentative, découvrent la ville qui deviendra leur ville d'inscription de deux façons possibles. Le premier mode d'accès à la connaissance d'un lieu se réalise en se rendant directement sur place : les vacances, les voyages, les visites à des amis apparaissent comme autant d'opérateurs de découverte du territoire. Le développement accru des mobilités ainsi que la diminution du temps de travail laissent augurer, dans cette perspective, d'une multiplication à venir des occasions de découverte. Le second mode d'accès à la connaissance d'un lieu s'effectue principalement à partir d'un média: lecture d'un livre dont l'action dévoile progressivement les caractéristiques d'un endroit donné, écoute d'un récit ou visionnage d'un film mettant en scène un lieu parmi d'autres; l'imaginaire lié au territoire constitue un motif de déplacement quel que soit le capital culturel et économique dont dispose le migrant. À l'horizon de tout déplacement n'y a-t-il pas l'espérance d'une vie meilleure? Comme le souligne G. Simon : "Il n'y a pas d'émigration volontaire intense ou nombreuse sans un imaginaire individuel ou collectif bien rempli, nourri de rêves et de visions heureuses, de chimères, voire de «châteaux en Espagne» » (Simon, 1998).

Dans les deux cas, ce déplacement qui s'apparente sur de nombreux points à un déplacement affectif et cognitif, renvoie à un véritable processus de cristallisation, une rencontre sociale marquant la rencontre entre un individu et un lieu et scellant ce moment privilégié où le migrant décide de s'y installer durablement. Ce déplacement "ultime mais non définitif» (Jordi, 2002) vers la ville d'inscription, parce qu'il s'apparente à une forme de désaffiliation ${ }^{4}$ (Castel, 1990), se traduit par l'ouverture du champ des possibles pour ces migrants volontairement partis et éclaire la relation complexe qu'ils entretiennent à l'égard du territoire.

Nous nous proposons donc d'examiner ici les formes que peut prendre la désaffiliation de façon à montrer, à partir de l'analyse de cette notion, combien la thématique du retour déborde largement ses prémisses géographiques. L'expérience du retour ne se limite pas en effet à l'acte de revenir physiquement sur des lieux laissés par choix ou par nécessité. Le retour renvoie également à l'expérience de retrouver des éléments familiers dans des lieux inconnus, des lieux où le migrant n'a jamais vécu. Parce qu'il interroge les qualités d'hospitalité inscrites dans les espaces traversés et qu'il réintroduit de la proximité dans l'éloignement, nous qualifierons ce retour de proxémique $^{5}$. Nous examinerons donc à la lumière de ce jeu entre retour physique et retour proxémique les conditions qui précèdent le départ et la découverte des villes d'inscription. Les indices de proximité entre lieux de départ et lieux d'inscription nous conduiront ensuite à inverser le sens du retour car il s'agit moins du retour physique que du retour proxémique qui importe pour comprendre l'expérience de la migration volontaire. 


\section{Départ imposé et départ souhaité}

7 Parmi les occurrences de la migration, s'opposent deux modalités principales de départs : départ imposé et départ souhaité. Nombreux sont en effet les récits de migrants dont celui des ruptures qui marque les trajectoires biographiques et rythme les histoires collectives. Partir renvoie alors à l'expérience du détachement de l'individu à l'égard des ressources matérielles, relationnelles et culturelles présentes dans un endroit donné.

8 La migration volontaire est, en effet, parfois précédée de l'expérience de l'exil et fait suite, comme nous le verrons, à une première migration non voulue, non désirée. Terme polysémique par excellence, la migration recouvre ainsi des expériences multiples, variées, parfois opposées, toujours renouvelées. Quand elle est le résultat d'un contexte politique et/ou économique défavorable et qu'elle s'affirme dans sa nécessité, le choix du pays de destination mobilise, bien souvent, différents paramètres tels les conditions d'accès, un réseau de connaissance, l'espoir d'un travail. Avant de connaitre l'expérience de la migration volontaire, les migrants se rendent le plus souvent soit vers le pays dont ils possèdent la nationalité, mais où ils n'ont pour la plupart jamais vécu (enfants d'expatriés ou vivant dans le pays depuis des générations) soit vers le pays qui, au niveau historique, a entretenu des liens particuliers avec le pays d'origine (pensons, par exemple, aux habitants de la Côte d'Ivoire ou des pays du Maghreb vers la France). Les plis singuliers de l'histoire dont chaque migrant est porteur confluent ainsi avec les plis forgés par l'histoire des déplacements en tout genre.

9 Ce déplacement contraint où le sentiment d'exil et de déracinement l'emporte dans un premier temps sur toute autre considération ne doit pas occulter l'autre occurrence de la migration, celle délibérément consentie qui n'est pas précédée, comme dans les cas précédents, par l'expérience de l'exil. Partir vers d'autres horizons apparait comme une solution possible quand la volonté de tourner la page et d'élargir son horizon s'impose comme une évidence, un acte autonome dont le principal ressort est le défi. Partir pour se réinscrire ailleurs se fonde alors sur une obligation que l'on se donne à soi-même, obligation qui se réalise certes dans un contexte d'incertitude, mais non de crainte immédiate et concrète pour sa propre existence. Il reste que bien souvent, des situations familiales douloureuses, telle la perte d'un proche par décès, précipitent la décision de migration dans un contexte où rien ne la laissait présager. Pour autant, même si les candidats à la migration partent volontairement, en dehors de toute contrainte économique, écologique ou politique, ils doivent se préparer à l'apprentissage de la langue du pays d'accueil, penser à réunir préalablement la somme d'argent nécessaire au voyage et indispensable lors des premiers temps de l'installation, rassembler, enfin, les nombreux justificatifs liés aux démarches administratives à entreprendre. Autant d'étapes incontournables avant le départ qui offrent finalement la possibilité de tester sur la durée le choix de la migration, a fortiori quand celle-ci s'affirme comme volontaire.

10 Indirecte ou directe mais non épargnée par les difficultés qui en résultent, la migration volontaire s'accompagne en outre d'un certain nombre de tributs dont la solitude, parfois le déclassement professionnel et le plus souvent l'incompréhension des membres de l'entourage restés sur place ne laissent entrevoir que les traces les plus saillantes. Le défi dont l'importance se mesure à travers les obstacles rencontrés et les 
difficultés surmontées est au cœur de ce type de migration. C'est en effet parce qu'ils font face à l'inconnu dans le pays d'accueil, parce qu'ils relèvent les gageures qu'ils se sont eux-mêmes imposés, que les migrants se constituent en sujet de leur propre histoire.

À l'intersection de ces deux types de migrations, on retrouve cependant une même épreuve fondatrice, celle de la déception. Cette déception que l'on peut présenter comme un « élément central de l'expérience humaine » (Hirschman, 1983) se traduit par un double mouvement de repli et d'ouverture : repli vers la sphère privée ou intime quand les migrants fuient un pays en proie à une situation politique difficile. L'isolement social accompagne alors bien souvent l'expérience de l'émigration dans le pays d'accueil. Ouverture et/ou élargissement à d'autres dimensions que les sphères familiale et/ou professionnelle quand le migrant décide de chercher dans l'ailleurs les conditions d'un renouveau. Ni la constitution d'une famille, ni la réalisation professionnelle ne permettent dans ces cas de trouver ou de retrouver les conditions de son accomplissement (De Gourcy, 2004). Le plus souvent, les migrants volontaires partent seuls en établissant néanmoins une mise à distance contrôlée des anciennes appartenances.

\section{Des parcours migratoires et des villes d'inscription}

La découverte de la ville d'inscription est une expérience décisive dans l'itinéraire biographique du migrant puisqu'elle déterminera la suite de son parcours. L'attrait des villes où se cristallise une rencontre entre les individus et des lieux tient pour une large part à la dimension de familiarité dont elles sont investies par ces derniers. Cette familiarité s'exprime de façon privilégiée à travers des indices de proximité qui donnent à voir des éléments paysagers de la ville d'inscription sur le mode de la ressemblance avec les paysages des lieux de l'origine. Cette " organisation perceptive " témoigne de la "relation dialogique qui s'instaure entre un espace urbain et celui qui le traverse, le parcourt ou l'explore " (Joseph, 1998). Se joue, en effet, dans ce type de relation entre l'homme et l'environnement, un processus associatif, une grammaire de l'intelligence iconique ${ }^{6}$ (Ferry, 2004) qui mobilise le vécu subjectif de l'individu et l'interpelle dans sa capacité à reconnaître puis à traduire ces indices de proximité selon les termes d'une historicité qui repose, le plus souvent, sur une mémoire familiale transmise des lieux découverts. Les aires géographiques, devenues les points de confluence de ces parcours migratoires, ne sont pas les villes de retour au sens physique et géographique du terme, mais les migrants les décrivent comme s'il s'agissait de lieux connus : à la jonction entre leur passé et leur présent, ils y retrouvent en effet des éléments de familiarité leur rappelant le pays quitté. Ce ralliement à ces lieux perçus comme familiers est d'autant plus prégnant qu'ils connaissent l'expérience de l'exil ou qu'ils n'ont pu trouver dans leur pays d'origine les ressources permettant de réaliser, de façon positive, l'« expérience de soi » (Ion, 2001).

Des horizons lointains sont laissés afin de se réinscrire ailleurs, dans un pays, une ville qui donnent la possibilité de retrouver les traces d'un passé vécu ou transmis. C'est à cette condition que s'efface le sentiment de déracinement et se pense l'acte d'inscription dans une ville qui fera sens pour le migrant. Tel est le cas de Geneviève qui a quitté l'Algérie, son pays natal, au moment de l'Indépendance pour arriver en France vers l'âge de vingt ans, dans un pays dont elle possède la nationalité mais où elle 
n'a jamais vécu, à l'exception de quelques congés, dans sa jeunesse, pour retrouver la famille laissée au pays. Fonctionnaire au sein de l'Éducation Nationale, elle s'est d'abord laissée guider pendant une dizaine d'années au gré de mutations successives dans des villes du nord de la France car, comme elle le dit elle-même, elle n'avait nulle part où aller. Cependant, une visite rendue à un ami habitant la Provence décide de la suite de son itinéraire résidentiel: la découverte de la ville d'Aix-en-Provence constitue, en effet, un moment décisif dans son parcours puisqu'elle choisit de s'y faire muter, au risque de perdre de nouveau un réseau relationnel reconstitué depuis son arrivée. Cette ville s'oppose à l'indistinction des autres cités françaises, car elle éveille chez elle les souvenirs de son enfance : "C'est une ville qui me plaît. Voilà, comme je vous dis, jusqu'à ce que je vienne à Aix, j'avais pas d'endroits où j'avais envie d'aller ", précise-t-elle.La perte de ce qu'apportait le pays natal est désormais compensée par les abords perçus comme familiers d'une ville devenue point d'ancrage :

"Alors il y a une chose à Aix qui est aussi, que j'aime beaucoup c'est la Sainte-Victoire parce que quand j'étais gosse, mes parents avaient une ferme en Algérie et à côté de cette ferme il y avait une montagne qui n'était pas si grande que Sainte-Victoire mais qui était exactement ce type de montagne, c'est-à-dire c'était un bloc rocheux comme ça qui s'appelait le Rocher des Pigeons et je crois que Sainte-Victoire me rappelle cet endroit que j'aimais beaucoup. Et donc, je me dis: "Oh, ben j'ai retrouvé quelque chose comme ça" ".

14 Fondatrice d'un nouveau rapport aux lieux (et souvent au temps), cette découverte va bien au-delà de l'élargissement des territoires connus, elle marque un nouveau départ dans l'itinéraire biographique des individus, une opération d'ajustement entre ce qui a été laissé et ce qui semble retrouvé. En se rendant le plus souvent par hasard dans une des trois villes qu'ils ne connaissaient pas, les migrants identifient des éléments perçus comme familiers (Schütz, 1994). Cette connaissance familière, que nous distinguerons d'une connaissance compétente ${ }^{7}$, permet une appréhension immédiate du monde environnant opérant par un changement de cadres: la découverte de la ville s'accompagne en effet de la transposition de cadres connus (Goffman, 1991) sur des cadres non connus, mais perçus dans leur familiarité.

La sécurité ontologique (Giddens, 1994) qu'apporte l'expérience de retrouver dans l'ailleurs ce qui a été perdu ou laissé en d'autres lieux exprime ses effets dans un autre témoignage, celui de Sara. Née à Sfax, en Tunisie, dans une famille juive établie depuis des générations, elle quitte son pays natal vers la vingtaine, n'y retournant que pour de rares et courts séjours. Contrairement au témoignage précédent, ce ne sont pas les circonstances politiques qui sont à l'origine de ce départ mais l'impossibilité, étant femme et intellectuelle, de projeter une carrière professionnelle dans ce pays. C'est à Marseille, ville découverte en allant rendre visite à des amis après le décès accidentel de son fils, qu'elle trouve une forme de compromis, une " réparation partielle de ce à quoi elle a été arrachée » selon ses termes. Le sentiment d'exil, ressenti depuis le départ de son pays natal, disparaîtra après avoir pu renouer avec les codes de sociabilité, liens ténus mais infrangibles qui la lient à son pays d'origine et désormais à son pays d'adoption. Les indices de proximité entre les deux pays, entre les deux villes, s'évoquent ici sous la forme d'un mode de vie, miroir commun des qualités expressives de l'être-ensemble :

"Les maisons ressemblent beaucoup à celles de Tunis de mon enfance si vous voulez. Quand je montais à Tunis qui a le même genre de volets, les gens trouvent normal qu'on vive avec les volets fermés pour tamiser la lumière. Et il y a une sociabilité comme je l'avais connue làbas où on plaisante beaucoup. Alors que j'avais continué à plaisanter dans le nord de la France, enfin à Paris et dans l'Yonne et dans la Mayenne mais personne ne comprenait. Donc 
on me traitait comme une espèce de caractérielle alors qu'ici il y a tout de suite quelqu'un

qui saisit et qui rigole, voilà. Je me sens beaucoup plus pouvoir être comme je suis ». rappelle que même éloigné dans le temps et l'espace, un endroit peut toujours faire partie, selon les termes de G. Perec, d'une « mémoire potentielle, d'une autobiographie probable» (Perec, 1995). Les paysages, les senteurs, une ambiance, les codes de sociabilité, autant de dimensions sensibles et perceptives d'un lieu qui témoignent du fait que la rencontre entre un individu et un endroit donné mobilise d'abord et avant tout les ressorts de la mémoire.

Expérience mémorielle et impression de renouveau accompagnent alors la découverte de la ville. Étape qui reste décisive dans le parcours du migrant puisqu'elle décide de son inscription résidentielle. Tel est le cas de Paquie, migrante d'origine espagnole, qui trouve dans Montpellier et ses entours des éléments familiers de son enfance passée en Espagne. L'installation dans cette ville atteste d'une forme de compromis entre ce pays des origines quitté pour des raisons économiques et le pays d'adoption où elle vit. La découverte de la ville s'est faite fortuitement mais la familiarité qui se manifeste dans ses entours apparaît ici comme une dimension de l'accueil et rappelle combien, entre le proche et le lointain, les échelles de mesure s'affranchissent des conventions collectives pour entrer dans un régime d'appréciation personnelle :

«C'est pas la ville, la ville ça ressemblait pas du tout. C'est les extérieurs. C'est les figuiers,

les amandiers, le paysage, si tu veux, surtout le paysage et puis le soleil ».

Ces indices de proximité, qui marquent la convergence entre ce qui a été perdu et ce qui est retrouvé, rappellent à ces migrants une époque révolue, évoquent des moments d'une enfance passée dans un cadre familier. Cette familiarité, ce souvenir des éléments connus, constituent des dimensions de l'accueil et confortent les migrants dans leur décision de rester. C'est à ce titre-là que l'on peut parler de retour car les migrants retrouvent, dans les villes rencontrées, des éléments familiers leur permettant de reconstituer un cadre propice à la définition d'un acte de fondation. Par-delà la distance, une même grammaire cognitive, élaborée à partir d'une syntaxe commune, réunit ces endroits géographiquement très éloignés. En outre, contrairement au pays d'origine, l'espace de la ville rencontrée, qui se prête à cette lisibilité immédiate, n'est pas associé à une mémoire douloureuse, aux circonstances politiques difficiles qui ont été à l'origine du départ - et parfois même de la fuite - du pays natal (migration volontaire indirecte).

\section{L'inversion du retour}

19 «Avant de quitter le Danemark, il s'était représenté le face-à-face avec les lieux connus, avec sa vie passée, et s'était demandé : serait-il ému? froid ? réjoui ? déprimé ? Rien de tout cela. Pendant son absence, un balai invisible était passé sur le paysage de sa jeunesse, effaçant tout ce qui lui était familier ; le face-à-face auquel il s'était attendu n'avait pas eu lieu " (Kundera, 2003). On peut se demander, dans ce contexte, quel(s) rapport(s) les migrants entretiennent avec les lieux de l'origine, les endroits d'où ils sont partis, après avoir fait l'expérience de cette rencontre affective et mémorielle avec l'une des villes d'inscription. Cette question se pose en effet puisque les migrants reviennent parfois lors de séjours de durée variable dans le pays ou la région d'origine. Il n'est d'ailleurs pas aisé de retourner (et se retourner) sur les traces de son passé, car

Revue européenne des migrations internationales, vol. 23 - n² | 2007 
dans ces retours sans cesse différés se joue la confrontation avec son histoire. Josef ou Irina, les personnages du roman de Kundera, seraient-ils revenus dans leur pays natal s'ils n'avaient pas été encouragés par leurs proches? Retourner ne signifie donc pas simplement refaire le chemin en sens inverse, c'est aussi une confrontation qui attend les migrants.

Signe de ces difficultés, tout semble se passer comme si l'expérience de la rencontre sociale avec les villes devenues villes d'inscription perturbait les anciens repères, les premiers jalons : lorsqu'ils retournent sur les lieux d'où ils sont partis, l'expérience du décalage est alors celle qu'ils vivent le plus souvent. Le décalage éprouvé dans le retour permet de mesurer le cheminement accompli, même si c'est au prix d'une division: être là sans y être, sans y retrouver ses repères familiers. Ce décalage qui rend le natif étranger en son pays n'est pas propre à ce type de migration puisqu'on le retrouve dans les migrations dites de nécessité où la contrainte de départ était forte. On rejoint donc ici l'expérience de l'immigré telle qu'elle est évoquée en France en 1975 par un ouvrier kabyle: "J'ai découvert ce qu'est l'exil [elghorba]. Ils ont beau plaisanter, quand ils reviennent au pays, sur "la terre natale qui leur est devenue la terre étrangère [elghorba]", l'exil c'est toujours l'exil. Ils disent bien: "Le pays m'est devenu l'exil [elghorba]", quand ils sont "pris dans l'obscurité", mais au fond, on ne les croit pas » (Sayad, 1999).

21 Le retour - au sens physique et géographique du terme - pose la question de l'hospitalité de celui qui prétend, malgré l'absence, être chez soi. Mais est-on vraiment chez-soi quand on ne reconnaît plus les lieux de l'origine tel Ulysse lorsqu'il accosta son Ithaque natale? Cette "aventure polysémique» qu'est le retour ouvre de façon frontale le questionnement sur le sens du chez-soi, de l'être chez-soi (Montandon, 1999), invite également à penser de manière renouvelée les formes de la reconnaissance dans le double sens de reconnaître et être reconnu. Cette double épreuve paraît fondamentale dans le procès de rebroussement mais reste une étape délicate - dont la littérature se fait l'écho - pour celui ou celle qui est devenu autre au fil du temps et des déplacements.

Aussi, de la tension entre l'espace d'origine et l'espace d'accueil résulte souvent un point de non-retour: le retour impossible. Ce processus au terme duquel le retour devient impossible, parce qu'improbable ou trop difficile, s'effectue souvent, d'ailleurs, à l'insu du migrant.

23 Quand il a lieu, il transmue le natif en observateur de son propre pays à travers le regard déplacé et décalé qui est désormais le sien. Car ce qui semble fondamental dans ce type de déplacement, c'est que les migrants reviennent dans leur pays d'origine avec un autre regard, ils sont porteurs d'une nouvelle expérience ${ }^{9}$, celle du renoncement, et le retour dans le pays d'origine se fait désormais à partir de la ville rencontrée. «Si je pars, c'est en tant que marseillais ", précisera un des migrants.

Comprendre cette «inversion du retour » suppose de tenir compte de l'élargissement du territoire circulatoire du migrant qui prend désormais en compte, outre les espaces fondateurs, soit les « lieux de la mémoire vive du migrant comme les lieux de l'enfance, de l'adolescence ", et les espaces de référence renvoyant "à l'ancestralité, aux lieux de l'origine familiale, à la mémoire historique non nécessairement vécue » (Gotman, 1999), un troisième endroit: ce dernier, très proche mais ne recouvrant pas forcément les deux précédents, est l'espace de la ville rencontrée où le migrant retrouve et convoque des éléments définis comme familiers et perçus dans leur immuabilité. Élargissement et 
transposition témoignent du changement d'échelle qui s'est opéré dans le rapport au monde du migrant et rappellent combien le rapport à l'espace s'inscrit moins dans le cadre d'une conception fixiste de l'appartenance, comme le laissent entendre les métaphores du déracinement, que dans le cadre d'une relation dynamique et inventive, un acte volontaire d'adhésion. Aussi, dans la relation aux lieux, se joue bien autre chose qu'une appartenance qui serait donnée par la naissance car l'horizon de référence n'est pas tant le pays natal, que celui où se réalisent les apprentissages ${ }^{10}$.

La découverte de cet endroit où s'opère cette tension entre le connu et l'inconnu renvoie ici à la dimension proxémique du retour. Cette dimension proxémique se traduit concrètement dans les dires des migrants par un discours de la réconciliation : contrairement au corps souffrant du migrant exilé, le corps du migrant volontaire est régénéré. On notera, dans cette perspective, l'importance des métaphores de la renaissance lesquelles traduisent les perspectives nouvelles qui s'offrent aux migrants. Un témoignage, celui d'Hélène, née en Algérie, qui a vécu au moment de l'Indépendance une tentative d'enlèvement alors qu'elle avait une douzaine d'années, illustre les effets régénérateurs de son établissement dans la ville rencontrée. Désormais établie à Montpellier après avoir vécu une dizaine d'années à Istanbul, l'installation dans cette ville a effectivement coïncidé avec la levée de ses inhibitions et des rituels de précaution dont elle s'entourait depuis des années. L'attachement à la ville d'inscription, l'engagement dans l'action lui ont permis de s'affranchir du poids de l'expérience vécue dans le pays d'origine et de trouver, grâce à cette désaffiliation consentie, les conditions de son accomplissement :
«Il y a des tas d'inhibitions qui ont disparu chez moi. Bon, je n'ai plus du tout peur dans la rue alors que pendant longtemps le traumatisme de mon enlèvement, si tu veux, au moment de l'Indépendance, je veux dire le soir quand je rentrais, je préparais ma clef avant de sortir de ma voiture. Je préparais toutes mes clefs tu vois, je sortais de la voiture, je me précipitais au portail, j'ouvrais et puis je fermais et tout. Je regardais à droite à gauche. Je n'ai plus du tout, je ne vis plus du tout de la même manière. Je n'ai plus du tout cette peur. [...] Alors moi je pense que ça vient depuis le fait que j'ai décidé de tout quitter comme ça et de vivre ma vie parce que je me suis dit: "Ça y est maintenant j'ai fait ce que j'avais désiré faire vraiment, réellement. J'ai réussi à le faire, je l'ai fait. Maintenant il peut m'arriver n'importe quoi, ça m'est égal" ".

Quand le retour dans le pays d'origine est, pour diverses raisons, impossible, trop difficile (perte des repères...) ou tout simplement non souhaité, les lieux de la rencontre sociale donnent la possibilité d'expérimenter une forme de retour grâce aux éléments familiers, perçus comme tels, que les migrants y retrouvent. Par cette familiarité qui constitue une dimension de l'accueil, les migrants peuvent désormais envisager de jeter les jalons pour de nouvelles attaches territoriales dans des endroits dépourvus de tout souvenir renvoyant à un passé douloureux (perte d'un proche, événements politiques contraignant à l'exil...). Attaches territoriales qui feront sens aussi pour les descendants du migrant car, dans le renouvellement de ce processus au fil des générations, le territoire de la rencontre sociale devient espace fondateur, parfois même espace de référence. À la croisée des qualités d'hospitalité et d'habitabilité, cette familiarité exprimant la jonction entre un passé et un présent atteste également du fait que l'on habite autant avec son corps qu'avec sa mémoire.

Le retour, entendu ici au sens de proxémique, n'est plus seulement un procès de déplacement, il témoigne alors du dé-placement affectif et cognitif qui caractérise 
l'expérience du migrant puisque, pour le dire autrement, il ne transporte pas (ou plus) une absence (Sayad, 1999) mais reconstitue, en de tels endroits, une présence.

\section{Installation et réparation}

déménagement, un changement de résidence ou encore un déplacement ne sont pas seulement des séquences à partir desquelles il est possible de reconstituer l'itinéraire résidentiel d'un individu donné, elles doivent être replacées dans l'expérience de vie du migrant pour en comprendre la totalité signifiante. La possibilité d'éprouver les conditions d'un renouveau au moment de la rencontre sociale renvoie désormais les déplacements précédant l'établissement dans la ville rencontrée dans l'oubli d'une époque où rien de significatif ne semble se détacher, expérience du temps dont la durée s'annule dans l'interchangeabilité des lieux et des événements. Cette déconstruction/ reconstruction de la période suivant le départ du pays d'origine jusqu'à l'établissement dans la ville rencontrée n'est pas le seul fait des migrants contraints de quitter le pays natal. Dans le cas de ceux qui ont migré volontairement et directement, on remarque que ce ne sont pas tant les expériences résidentielles qui semblent non significatives que la trame d'une existence devenue sans surprise: la lassitude, l'insatisfaction l'emportent alors dans le récit descriptif et narratif de leur mode de vie.

Qu'ils se soient déplacés par choix pour fuir un horizon social devenu trop fermé ou qu'ils aient été contraints au déplacement avant de migrer ensuite volontairement vers les villes rencontrées, dans les deux cas, les migrants retrouvent une place ${ }^{11}$ qui, progressivement, parvient à leur faire oublier l'expérience communément partagée de la déception. Car au-delà de ce que la familiarité apporte, il s'agit de renouer les fils d'un itinéraire biographique ${ }^{12}$ sans pour autant occulter ou "refouler » le passé perçu comme douloureux ou difficile. C'est à cette condition que s'ouvrira leur «horizon d'attente ", cet horizon d'attente que nous définirons à la suite de R. Koselleck comme ce qui « s'accomplit dans le présent et est un futur actualisé, elle [l'attente] tend à cequi-n'est-pas-encore, à ce-qui-n'est-pas-du-champ-de-l'expérience, à ce-qui-n'estencore-qu'aménageable » (Koselleck, 1990). Cette ouverture des perspectives permet le redéploiement sur le temps de l'action, sur le présent de la réalisation. Loin de toutes considérations ou préoccupations d'ordre climatique, comme pourrait le laisser penser de prime abord le choix de ces destinations ensoleillées, l'établissement dans les villes rencontrées apporte avant tout cette sécurité à laquelle les migrants aspirent, cette confiance nécessaire à la redéfinition de leur horizon d'attente.

La migration volontaire, qui vient bien souvent solder un parcours empli de déplacements, donne alors l'occasion de «refermer la boucle » par l'expérience de la découverte d'un lieu qui fait sens au regard de l'histoire de vie du migrant. N'y a-t-il pas là une forme de réparation qui s'apparente également à une forme de réconciliation avec une histoire de vie marquée par l'expérience de la rupture? Revenons, pour conclure, sur un ultime témoignage, celui de Sara désormais installée - et, semble-t-il, durablement implantée - dans la cité phocéenne. L'expérience de la continuité dont elle nous fait part se manifeste ici comme un élément structurant de l'existence et rappelle combien le sentiment d'appartenance tient parfois moins à un espace de référence ou à un espace fondateur qu'au fait de retrouver une proximité que nous qualifions d'analogique entre les lieux. Cette continuité n'apparaît donc pas seulement comme une donnée liée à une appartenance aux lieux fondateurs, elle se révèle

Revue européenne des migrations internationales, vol. 23 - n² | 2007 
également dans les villes d'inscription grâce au regard porté sur les lieux de l'origine, ce que l'on en retient et transpose ensuite dans la qualification des espaces :

«Tous les gens comme moi qui ont été coupés de leur communauté d'origine par un hasard historique, comme l'Indépendance de la Tunisie pour les Juifs, voyez, ou bien l'Indépendance de l'Algérie pour les petits Blancs, pour des petits Français. Ceux-là ils ont forcément quelque chose à refaire et à trouver. On considère, on passe ça par profits et pertes parce que c'est pas grave comme la Shoah ou que tout le monde n'est pas mort, qu'on n'a pas vécu des sévices atroces. Mais en même temps il y a une perte réelle de quelque chose qui contentait parfaitement. Donc une série de gens qui appartiennent à la même catégorie que moi vont inévitablement se retrouver dans ces choix de vivre à Marseille, de vivre en Provence, à Montpellier, voyez, c'est inévitable. Il s'agit de reconstituer une continuité, enfin de renouer avec une continuité à laquelle tous les êtres humains ont droit mais qu'ils ont en général sans se poser de questions. Et c'est pas la même chose d'être un Français du nord qui avait choisi de venir à Marseille parce qu'il a horreur de la pluie que d'être quelqu'un qui est né dans un climat encore plus plaisant que celui-là et qui trouve là une réparation partielle de ce à quoi il a été arraché ".

\section{En conclusion}

Pour bénéficier de cette sécurité qu'apporte le sentiment d'inscription, les voies sont nombreuses. Leur nombre diminue cependant quand elles sont parsemées d'écueils et d'embûches. Parmi ces écueils, nous avons envisagé les ruptures dans les itinéraires biographiques : ruptures induites par un contexte politique défavorable contraignant à l'exil ou ruptures manifestées dans la volonté de s'affranchir des paramètres d'une vie devenue insatisfaisante et d'élargir ainsi son horizon d'attente grâce à la migration. Ces situations de rupture qui marquent l'expérience de la désaffiliation - négative dans le premier cas, positive dans le second - et qui projettent les migrants sur la route des errances trouveront leur accomplissement dans la possibilité d'une réaffiliation. Cette réaffiliation qu'induit la fixation sur un lieu dépourvu de tout rappel douloureux mais évoquant, à travers différents paramètres, les dimensions du souvenir est la condition de leur redéploiement, de l'ouverture de leur horizon d'attente. La grammaire iconique (Ferry, 2004) qui s'appuie sur l'histoire privée des individus et lui confère sa valeur indiciaire leur permet de (re) trouver les conditions d'une sécurité ontologique tout en donnant un sens à la migration volontaire et en élargissant ainsi les possibles contenus dans la notion de retour. Saisir la notion de retour sur le seul plan géographique paraît donc insuffisant pour en comprendre les multiples aspects, il faut tenir compte des dimensions esthétique, émotive et cognitive qui se jouent dans ces espaces/temps. Le retour, dans cette perspective, ne renvoie plus à un déplacement qui s'exprime en sens inverse du précédent car le territoire circulatoire du migrant s'est élargi : il tient désormais compte de l'espace de la ville rencontrée, cet endroit qui rend possible l'invention d'une vie autre. 


\section{BIBLIOGRAPHIE}

CASTEL Robert (1970) Le roman de la désaffiliation, Le débat, 61, pp. 152-164.

DE GOURCY Constance (2005) L'autonomie dans la migration. Réflexion autour d'une énigme, Préface de M. MARIÉ, Paris, L'Harmattan, 347 p.

DE GOURCY Constance (2004) D'un lieu à l'autre : les femmes dans le processus migratoire, in Sylvette Denefle Éd., Femmes et villes, Tours, Presses Universitaires François-Rabelais, pp. 513-527.

FERRY Jean-Marc (2004) Les grammaires de l'intelligence, Paris, Cerf, 211 p.

GOFFMAN Erving (1991) Les cadres de l'expérience, Paris, Minuit, 573 p.

GOTMAN Anne (1999) Géographies familiales, migrations et générations, in Catherine Bonvalet, Anne Gotman, Yves Grafmeyer Éds., La famille et ses proches ; L'aménagement des territoires, Paris, INED-PUF, Travaux et documents, Cahier 143, pp. 69-133.

HALL T. Edward (1971) La dimension cachée, Paris, Seuil, 254 p.

HIRSCHMAN Albert (1983) Bonheur privé, action publique, Paris, Fayard, 257 p.

ION Jacques (2001) Affranchissements et engagements personnels, in Jacques Ion Éd., L'engagement au pluriel, CRESAL, Publications de l'Université de Saint-Etienne, pp. 22-45.

JAVEAU Claude (1991) La société au jour le jour ; Écrits sur la vie quotidienne, Bruxelles, De BoeckWesmael, $292 \mathrm{p}$.

JORDI Jean-Jacques (2002) Les pieds-noirs : constructions identitaires et réinvention des origines, Hommes et migrations, $\mathrm{n}^{\circ}$ 1236, pp. 14-25.

JOSEPH Isaac (1998) La ville sans qualités, La Tour d'Aigues, L'aube, 209 p.

KOSELLECK Reinhart (1990) Le futur passé; Contribution à la sémantique des temps historiques, Paris, Éd. de l'E.H.E.S.S., 334 p.

KUNDERA Milan (2003) L'ignorance, Paris, Gallimard, 181 p.

MONTANDON Alain (1999) Le retour d'Ulysse, in Rose Duroux et Alain Montandon, Éds, L'émigration : le retour, Clermont-Ferrand, Presses Universitaires Blaise Pascal, pp. 13-21.

PEREC Georges (1995) Ellis Island, Paris, p.O.L., 71 p.

SAYAD Abdelmaleck (1999) La double absence. Des illusions de l'émigré aux souffrances de l'immigré, Paris : Seuil, $437 \mathrm{p}$.

SIMON Gildas (1998) La planétarisation des migrations internationales, in Rémy KNAFOU Éd., La planète « nomade»; Les mobilités géographiques d'aujourd'hui, Paris, Belin, pp. 59-76.

SCHUTZ Alfred (1994) Le chercheur et le quotidien ; Phénoménologie des sciences sociales, Paris, Méridiens Klincksieck, 286 p.

\section{NOTES}

1. Notons toutefois que cette notion admet la possibilité de ne pas rebrousser chemin : la route sans retour renvoie à l'idée de disparition; on parle aussi de partir sans esprit de retour quand on n'envisage pas de revenir. 
2. Il s'agit d'Aix-en-Provence, Marseille, Montpellier.

3. Des récits en profondeur effectués auprès de quarante-trois migrants appartenant aux couches moyennes de la population et provenant d'horizons géographiques divers (Amérique, Asie, Europe) ont permis de s'intéresser autant au migrant lui-même (niveau intime de la migration) qu'à son histoire familiale (niveau historique) en envisageant en particulier, à travers le prisme de la longue histoire, les déplacements que son groupe familial avait pu mettre en œuvre dans le passé.

4. La désaffiliation sera ici entendue au sens où les migrants se placent délibérément en situation de décrochage par rapport aux déterminations économiques et sociales.

5. Dans «La dimension cachée », E.T. Hall définit la "proxémie» comme l'ensemble des observations et théories concernant l'usage que l'homme fait de l'espace en tant que produit culturel spécifique. Nous ajoutons à cette définition l'idée que la relation de l'homme à son environnement matériel et social est médiatisée par une relation de proximité/familiarité nécessaire pour percevoir des lieux à travers leurs dimensions d'accueil.

6. La grammaire iconique fonctionne sur la base d'association d'images. Sur cette grammaire, chaque individu dispose son histoire privée d'une façon qui n'appartient qu'à lui. Cette grammaire se saisit, au niveau des formes langagières, à travers des discours descriptifs qui ouvrent sur la connaissance/reconnaissance des lieux qui font sens pour l'individu.

7. La connaissance compétente implique en effet une "maitrise conceptuelle, éventuellement instrumentale, de l'objet connu » (Javeau, 1991).

8. Quand la migration volontaire fait suite à un premier départ forcé, on notera que la volonté de conserver ses souvenirs intacts se heurte au désir de revoir le cadre familier. Il en résulte une forme d'appréhension et un retour sans cesse repoussé, parfois accompli en présence des enfants pour qu'ils connaissent ces lieux de l'origine.

9. Si partir volontairement implique de renoncer à ce qui avait été entrepris dans le pays d'origine pour l'incertitude de ce que le migrant trouvera dans le pays d'accueil, on remarque, que rares sont ceux qui reviennent sans avoir rien réalisé sur le plan économique. Les bénéfices obtenus, mêmes minimes, jouent comme condition de légitimation du déplacement volontaire.

10. Outre ces apprentissages réalisés dans le pays d'accueil, il ne faut pas non plus sous-estimer le travail de l'oubli dans le rapport aux lieux de départ.

11. Au sens premier du terme, soit cet espace qu'occupe, que peut occuper ou que doit occuper une personne.

12. Cette expression a été préférée à celle de trajectoire biographique qui par le côté linéaire qu'elle laisse supposer intègre moins les ruptures et les bifurcations inhérentes à tout parcours biographique.

\section{RÉSUMÉS}

Cet article a pour objet l'examen des formes que peut prendre le retour dans le cadre de la migration volontaire. Souvent considéré à partir de prémisses géographiques, le retour ne se limite pourtant pas à l'acte de revenir physiquement sur des lieux laissés par choix ou par nécessité. Il peut en effet renvoyer à l'expérience de retrouver des éléments familiers dans des endroits qui ne le sont pas, des endroits où le migrant n'a jamais vécu. Ce retour que nous qualifierons de "proxémique » interroge les qualités d'hospitalité inscrites dans les espaces 
traversés et réintroduit de la proximité dans l'éloignement, condition nécessaire à l'inscription dans les villes d'accueil.

The Migrant's Return Viewed through its Detours, or: how to reintroduce Proximity into Remoteness. The goal of this article is to examine the potential forms of return to the country of origin after voluntary emigration. Although often viewed from the standpoint of geography, return does not merely consist in physically going back to the places one had left. It may involve reexperiencing familiar items in unfamiliar places one had never been dwelling in. This "proxemic" type of return questions the hospitality offered in these spaces, and reintroduces proximity -a sense of home- into remoteness, which is a necessary condition for taking root in the new location.

La vuelta del migrante, vista a través de sus desvíos, o: cómo reintroducir proximidad en la lejanía. Este artículo examina las formas posibles de la vuelta del emigrante voluntario. Aunque muchas veces se considera solamente de un punto de vista geográfico, la vuelta no se limita al acto de volver físicamente en lugares que uno dejó. Puede corresponder a la experiencia de reencontrar elementos familiares en sitios no familiares donde nunca vivió el migrante. Esta vuelta «proxémica» pone en juego la hospitalidad oferta en los espacios atravesados y reintroduce proximidad en la lejanía - condición necesaria para la (re)inserción.

\section{AUTEUR}

\section{CONSTANCE DE GOURCY}

Maître de conférences, Département de Sociologie, Université de Provence, 29 avenue Robert Schuman, 13621 Aix-en-Provence Cedex 1. 